\title{
Cyprian als Enthusiast.
}

Von A. Harnack in Berlin.

Eine Untersuchung über Cyprian fält an sich nicht mehr in den Kreis dieser Zeitschrift; aber "Cyprian als Enthusiast" darf sich in ihr doch sehen lassen; denn es ist ein Stück urchristlichen Altertums, das sich hier noch fortsetzt.

Wer von Tertullian zu Cyprian in den geschichtlichen Betrachtungen fortschreitet, glaubt in eine neue kirchengeschichtliche Periode eingetreten zu sein, die von der Tertullian's durch ein Jahrhundert getrennt sein müsse; und doch liegt zwischen beiden eine knappe Generation. Selten bieten in der Geschichte Lehrer und Schüler samt der Zeit, der sie angehören, ein so verschiedenes Bild!

Aber es finden sich doch bei Cyprian einige Züge, in denen er sich als ein Epigone der tertullianischen, ja einer noch früheren Epoche erweist - wunderbar genug bei dem Manne, der den Episkopat nicht nur zum Kerne der Christenheit, sondern auch zur Grundlage der Kirche gemacht hat, und der an Dutzenden von Stellen schreibt wie ein mittelalterlicher Papst. Seine Haltung im Ketzertaufstreit gehört hierher, in noch höherem Masse seine - das Hysteron-Proteron sei gestattet donatistische Gesinnung (ep. 65. 67). Durch beides hat er seinem grossen Verehrer Augustin und der gesamten nordafrikanischen Kirche des 4. und 5. Jahrhunderts nachträglich die peinlichsten Schwierigkeiten bereitet. Dass er nicht selbst um allen Kredit gekommen ist, verdankt er dem Gedächtnis an seine geschichtliche Grösse, seinem Martyrium und seiner unvergleichlichen Bedeutung als abendländischer kirchlicher Schriftsteller.

In der "Altertümlichkeit", die er im Ketzertaufstreit und gegenüber lasterhaften Bischöfen bewährt hat, ist er durchweg ehrlich: was er hier zum Ausdruck gebracht, war ihm Herzenssache, wenn auch sein Bischofs- 
und Kirchenbewusstsein in jenem Strcite sehr stark betciligt gewesen ist. Schwieriger ist es über scincn Enthusiasmus zu urteilen.

Cyprian cin Enthusiast? wer will das glauben! Und doch finden

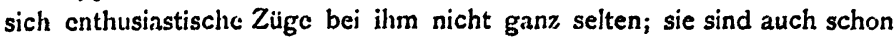
öfters bemerkt, aber $\mathrm{m}$. W. im Zusammenhang und in ihrer Eigenart nicht geniigend gewürdigt worden.

I.

Zum erstenmal lernen wir ihn als Visionär, d. h. als solchen, den Gott besondercr Offenbarungen gewürdigt hat, im I1. Brief kennen. Scine Situation war eine besonders ernste. Im Verstecke weilend, muss er für sein Ansehen in der Gemeinde fürchten, die durch die Verfolgung decimiert ist und durch die Heroen, die Confessoren, aufgeregt wird. Kaum zwei Jahre waren seit seiner Bischofsweihe verflossen; eine feste Stellung hatte er sich noch nicht erwerben können - seine WVahl war im Widerspruch zu einem Teile der Presbyter erfolgt -; in Rom war man seiner Flucht wegen von tiefem Mlisstrauen gegen ihn erfullt und hatte diesem Misstrauen auch in Karthago Ausdruck gegeben. Nun soll er, der Entflohene, die Schwachen stärken, den Feigen Mut einflössen, den Hochmut der Heroen zügeln, die Einheit der Gemeinde und die Ordnung aufrecht erhalten! In den Briefen 7. 5. 6. 8. 9. 13. I4. 12 [wahrscheinlich in dieser Reihenfolge] hat er dies mit Geschick und Würde gethan, dabei verschiedene Töne anschlagend, von denen er hoffen durfte, dass sie der Gemeinde und den Confessoren zu Herzen gehen werden; aber die Nachrichten, die aus Karthago kamen, waren nicht beruhigend. Er versuchte es nun mit einem sehr ernsten Schreiben: die Verfolgung und Verwüstung der Gemeinde. ist durch unsere Sünden hereingebrochen, weil wir den Weg des Herrn verlassen hatten, und nach Verdienst werden wir gerichtet. Unsre Habsucht, unser Hochmut, unsre Zwietracht und Eifersucht sind Schuld. Augenscheinlich dauerten diese groben Missstände auch während der Verfolgung fort. „Doch, welche Schläge, welche Hiebe verdienen wir nicht, da selbst die Bekenner, die doch den.anderen das Beispiel guter Sitten geben sollten, die Disciplin nicht beobachten! Da das aufgeblasene und unverschämte Prahlen mit dem öffentlich abgelegten Bekenntnis einige $z u$ frecher Überhebung geführt hat, so kamen nun die Martern - Martern, die nicht mit der Krone endigen, sondern den Bekenner dem natürlichen Tode überliefern." 
In den aufgeblasenen, zuchtlosen Bekennern also sieht er den Hauptfeind der Gemeinde und den schlimmsten Gegner. Mit Recht; denn wie sollte er ihm beikommen? Dass sich Gott zu ihnen bekannt, der heilige Geist ihnen das Bekenntnis auf die Lippen gelegt, Christus in ihnen Wohnung genommen hat, konnte er nicht leugnen. Wie ihnen begegnen? Der "geistliche Mensch" wird von niemandem gerichtet; sie aber waren ohne Zweifel ,geistliche" Menschen. Nur Ermahnen, Bitten, Anflehen war hier am Platze. Aber die Gemeinde musste ihrem Einflusse entzogen werden; freilich auch das war schliesslich nur mit dem Mittel des "Geistes" möglich; denn ohne denselben waren seine Waffen zu schwach. Dagegen Geist wider Geist - das hatte man schon oft erlebt, ohne daraus die Folgerung zu ziehen, dass zwei "Geister" sich gegenseitig aufheben, Cyprian fährt daher in seinem Schreiben an seine Gemeinde (c. 3) fort:

"Quod magis suasit et compulit, ut has ad vos litteras scriberem, scire debetis, sicut dominus ostendere et revelare dignatur, dictum esse in visione: ,Petite et impetratis', tunc deinde praeceptum plebi adsistenti ut pro quibusdam personis designatis sibi peterent, in petendo autem fuisse dissonas voces et dispares voluntates et vehementer hoc displicuisse illi qui dixerat: ,Petite et impetratis', quod plebis inaequalitas discreparet nec esset fratrum consensio una et simplex et iuncta concordia". [Cyprian geht nun wieder in die gewöhnliche Rede über, bringt Bibelsprüche, die Einheit betreffend, bei und schliesst den Abschnitt mit den Worten: „non venissent fratribus haec mala - die schweren Foltern statt der Märtyrerkrone oder der Freiheit -, si in unum fraternitas fuisset animata"].

Wer diese Vision erlebt hat, das sagt Cyprian nicht; die Ausleger nehmen hier und bei den gleich $z a$ besprechenden beiden folgenden Visionen als selbstverständlich an, dass Cyprian selbst der Visionär sei ${ }^{x}$; aber die 4. Vision .(c. 6) ist so eingeführt; dass man deutlich erkennt, Cyprian stützt sich in der I. (und in der 2. und 3. Vision) auf eine wunderbare Mitteilung, die andere (in seiner Umgebung) erhalten hatten ${ }^{2}$ (vgl. ep. 16, 4). Die Hauptsache bleibt, dass er sich auf die Vision gestützt hat. Diese selbst ist wenig durchsichtig geschildert, geht in eine biblische Admonition unmerklich über und zeigt auch dadurch, dass sie nicht aus

I In der deutschen Übersetzung (Kemptener Bibliothek) ist sogar ein "mir“ eingeschoben.

2 In der 2. Vision heisst es zudem ausdrücklich: „ille qui vidit"; dass sich Cyprian selbst hinter diesen Ausdruck rersteckt hat, ist eine grundlose Annahime. 
crster Hand mitgeteilt wird. Dic Scene ist etwa so zu denken: die Gemcinde ist im Gottesdienst versammelt, voll Sorge um das Geschick ihrer eingekerkerten Brüder; da crgeht eine Stimme: „Bittet, so werdet ihr empfangen"; dic Gemeinde wird demgemäss angewiesen, für diese cingckerkerten Brüder Fürbitte zu leisten (vielleicht nicht nur für sie, sondern auch für den im Versteck weilenden Bischof, damit er ihnen zurückgegeben werde: so crklärt sich der dunkle Ausdruck "pro quibusdam personis designatis sibi“, am besten); allein kein einmütiges Gebet, sondern verworrene und uncinige Stimmen dringen zu Gott herauf; natürlich kann er nun nicht helfen; denn solche Gebete missfallen ihm. Die Vision ist undeutlich - wo hat sie angefangen, wo endigt sie? wie verhält sich Vision und Interpretation? - aber die Tendenz ist unmissverständlich. Cyprian begnügt sich jedoch nicht mit dieser Mitteilung, die er geradezu als Veranlassung seines Briefs bezeichnet (,suasit et compulit"). Er fährt fort (c. 4):

„Nam et illud ostensuin est quod sederet paterfamilias sedente sibi ad dexteram iuvene, qui iuvenis anxius et cum quadam indignatione subtristis maxillam manu tenens maesto vultu sedebat. alius vero in sinistra parte consistens rete portabat, quod se mittere ut circumstantem populum caperet minabatur. et cum miraretur quid hoc esset ille qui vidit, dictum est ei iuvenem qui ad dexteram sic sederet contristari et dolere quod praecepta sua non observarentur, illum vero in sinistra exultare quod sibi daretur occasio ut a paterfamilias potestatem sumeret saeviendi. hoc prius longe ostensum est quam tempestas vastitatis huius oriretur. et videmus impletum quod fuerat ostensum".

Dies ist eine wirkliche Vision - ein einziges klares Bild - oder doch die glücklichste Nachbildung einer solchen. Aber noch mehr, es ist eine klassische Vision; denn sie wiederholt einen Urtypus der geistlichen Anschauung: Gott als Hausvater in der Mitte sitzend, ihm zur Rechten das gute Princip - hier natürlich Christus, in der gewöhnlichen Erscheinungsform als Jüngling - ihm zur Linken der Satan. Diese Vision geht in ihren Ursprüngen weit zurück, weit hinter Hiob, in die grauesten Vorzeiten der Religion. Der trauernde Christus, der das Kinn mit dem Arme stützt (also auch „Bein mit Beine deckt“) man beachte, dass sein Zorn und Unwillen aus Scheu nur leise angedeutet ist -, muss zusehen, wie sein böser Rivale, der mit dem Netz dasteht, von Gott die Gewalt zu wüten empfängt; denn wenn die Menschen die Gebote übertreten, so muss der Teufel sein Recht empfangen. Der Teufel zur Linken Gottes! in einem Zeitalter, in welchem 
bereits um dogmatische Kleinigkeiten (Monarchianismus) gestritten wurde, erbaute man sich noch an einem solchen Bilde, oder vielmehr, man sah es noch und zitterte. Dass die Interpretation in der Vision selbst erfolgt, ist etwas ganz gewöhnliches; der angelus interpres ist in der Regel zur Stelle. - Wiederum ist die Tendenz, in der Cyprian die Vision gebraucht hat, ganz unmissverständlich. Dass die Vision schon vor längerer Zeit sich ereignet hat, erhöht ihm ilhre Bedeutung; denn sie hat sich erfüllt: der Teufel fängt die Menschen und wütet in der Gemeinde. Es folgen nun im Briefe sehr eindringliche Ermahnungen zum Gebet, eingeleitet durch einen dritten Hinweis auf ausserordentliche göttliche Anweisungen (c. 5):

"Oremus instanter et adsiduis precibus ingemescamus. nam et hoc nobis non olim per visionem exprobratum est sciatis, quod dormitemus in precibus nec vigilanter oremus". Hier handelt es sich um Vorwürfe, die Gott in der Vision erhoben hat; aber auch diesmal ist noch nicht Cyprian selbst der Empfänger; denn also fährt er (c. 6) fort:

„Denique ad minimum famulum suum et in delictis licet plurimis constitutum et dignatione eius indignum tamen ille pro sua circa nos bonitate mandare dignatus est. ,dic illi', inquit, securus sit, quia pax ventura est, sed quod interim morula est, supersunt adhuc aliqui qui probentur. sed et de victu parco et sobrio potu divinis dignationibus admonemur".

Also nun erst fưhrt Cyprian eine ihm selbst gewordene göttliche Mitteilung ins Feld. Dass ihn, den entflohenen Bischof, Gott einer solchen gewürdigt hat, musste den Lesern auffallend sein $^{x}$. Cyprian durfte daher nur im Tone tiefster Bescheidenheit von ihr sprechen. Doch sind solche Äusserungen der Selbstwegwerfung schon längst bei Predigern üblich gewesen; man vgl. die Briefe des Ignatius ( $\pi \in \rho i \psi \eta \mu \alpha$ ú $\mu \omega ̂ v$ ) und die Homilie, die unter dem Namen „2. Clemensbrief" citiert

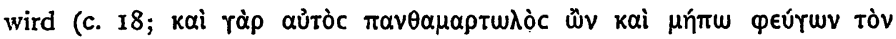

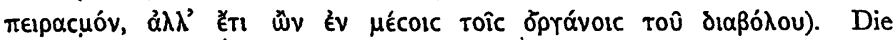
Vision ist so zu denken, dass Gott einem Engel - er ist der mit "dic" Angeredete - die Botschaft an Cyprian überträgt (vgl. den „Hirten“). Die Botschaft ist eine doppelte: der Friede kommt bald; aber noch müssen etliche erprobt werden - eine kluge Mitteilung, bei der die Thatsache, dass überhaupt etwas mitgeteilt wird, die Hauptsache ist. Die göttlichen Admonitionen gegen Völlerei scheinen nicht an Cyprian, sondern an andere ergangen $\mathrm{zu}$ sein.

\footnotetext{
I Doch hoffte Cyprian schon ep. 7 auf göttliche Anweisungen.
} 
2.

Der Bricf hat trotz des Aufgebots der stärksten Mittel seinen Zweck nicht erreicht. Dic Auflüsung der Gemeinde' machte Fortschritte, und leichtfertige und ehrgeizigc Confessoren untcrgruben durch ihre Friedensbriefe alle Zucht und Ordnung in der Gemeinde. Dic drei Bricfe 15-17 trcten diesem Treiben mit neuem Nachdruck entgegen. Daher werden auch wieder Visionen aufgeboten, und zwar, wie im I1. Brief, Visionen anderer und eine solche, die Cyprian selbst zu Teil geworden ist (cp. 16, 4):

"Castigare nos itaque divina censura nec noctibus desinit nec diebus. practer nocturnas visiones por dies quoque impletur apud nos spiritu sancto pueronum innocens aetas, quae in ecstasi videt oculis et audit et loquitur ea quibus nos dominus monere et instruere dignatur. et audietis omnia quando me ad vos reducem fecerit dominus, qui ut secederem iussit".

Das Bild, welches wir hier von der Stimmung der Umgebung Cyprian's im Versteck ${ }^{2}$ erhalten, ist sehr instruktiv. Dieser kluge, energische und herrschsiichtige Mann ist von Visionären und Ekstatikern umgeben. Nicht nur Nachts ereignen sich die Visionen, sondern auch am Tage, und ergriffen von der Ekstase werden vor allem die halbwüchsigen Knaben ${ }^{3}$, die sich um Cyprian befinden. (Es sind vielleicht dieselben, deren Visionen er im II. Brief erzählt hat; denn zwischen beiden Briefen liegt nur eine kurze Zeit). Der Ausbruch dieser visionären Erscheinungen kann nicht ohne Einwirkung, jedenfalls nicht ohne Zulassung, Cyprians selbst erfolgt sein; denn er beherrschte seine Umgebung. So sehr war er also trotz seines Klerikalismus ein Christ alten Schlages4. Aber ein doppeltes ist hier noch wichtig: erstlich, diesmal berichtet er nicht die Visionen selbst; er verschiebt vielmehr die Mitteilung bis auf seine Rückkehr; vielleicht soll darin eine Art Strafe liegen. Zweitens, er sagt diesmal mit dürren Worten, dass er auf einen göttlichen Befehl hin sein Bisthum ver-

I Erschütternd ist in dieser Hinsicht der Schlusssatz des I4. Briefes nach Karthago: „Fraternitatem si qua vobiscum est multum a me salutate".

2 .Wo dieses Versteck zu suchen ist, ist unbekannt.

3 Der Ausdruck "innocens aetas" stammt aus Tertull., de bapt.

4 In den römischen Schreiben, die sich in der cyprianischen Sammlung befinden, fehilt das visionäre Element ganz. Die relative Nüchternheit selbst der römischen Confessoren tritt in dem Brief $3 \mathbf{I}$ (c. 2) an Cyprian charakteristisch hervor: „Ex tuis litteris vidimus gloriosos illos martyrum triumphos et oculis nostris quadammodo caelum illos petentes prosecuti sumus et inter angelos .... constitutos quasi contemplati sumus. sed et dominum apud patrem suum testimonium illis promissum perhibentem auribus nostris quodammodo sensimus". Auch von Novatian wird niemals etwas Ekstatisches berichtet. 
lassen habe und ins Versteck gegangen sei. Dies $z \mathrm{u}$ behaupten hat er bisher nicht gewagt und hat auch die Behauptung niemals wiederholt? Sie wirkt eben in ihrer. Isolierung recht peinlich: wäre er sich wirklich bewusst gewesen, lediglich einem göttlichen Befehl gefolgt zu sein, so müsste der Ton seiner Briefe anders lauten als er lautet.

Als Cyprian nicht mehr länger zögern durfte, in seine Gemeinde zurückzukehren, obgleich die Verhältnisse sich inmer ungünstiger für ihn gestaltet hatten, beschloss er zuvor den Clerus mit Männern seines Vertrauens durch eigene Wahl zu besetzen. Das war ein kühnes Unternehmen, ja dem Herkommen gegenüber ein Gewaltstreich; aber er musste ihn riskieren. Die Wahl des Saturus und Optatus kündigt er dem Clerus einfach an (weil sie, wie er behauptet, schon früher zu diesen Ämtern designiert worden sein), die aber des Celerinus (ep. 39, I) und Numidicus (ep. 40, I) motiviert er unter Berufung auf göttliche Kundgebungen ${ }^{2}$. In bezug auf ersteren schreibt er nach Karthago:

"Celerinum fratrem nostrum ... clero nostro non kumana suffragatione sed divina dignatione (coniunximus). qui cum consentire dubitaret, ecclesiae ipsius admonitu et hortatu in visione per noctem compulsus est ne negaret. nobis suadentibus cui plus licuit [scil. deus] et coegit". In bezug auf letzteren:

"Admonitos nos et instructos sciatis dignatione divina, ut Numidicus presbyter adscribatur presbyterorum Carthaginiensium numero etc.“.

Man kann hier eine Steigerung beobachten. Die ersten beiden kündigt Cyprian einfach der Gemeinde an, weil er durch ihre Wahl nur eine frühere Designation verwirkliche; die Einsetzung des Celerinus wird durch eine Vision nachträglich von Gott bestätigt - Celerinus hat die Offenbarung gehabt - ; die Einsetzung des Numidicus wird auf direkte göttliche Anweisung zurückgeführt.

I In dem Brief an die Römer (ep. 20, 1) schreibt er: „Sicut domini mandata instruunt, orto statim turbationis impetu primo, cum me clamore violento frequenter populus flagitasset, non tam meam salutem quam quietem fratrum publicam cogitans interim secessi, ne per inverecundam praesentiam nostram seditio quae coeperat plus provocaretur". Das lautet anders; die "domini mandata" können nicht Spezialoffenbarungen sein (es würde wohl in diesem Fall der Singular stehen), sondern evangelische Sprüche. "Inverecunda praesentia nostra“ ist übrigens in diesem Fall ein recht dunkler Ausdruck.

2 Schon ep. 33, 2 hat er seinen Entschluss, gehorsame Gefallene milde zu behandeln auf Eingebung Gottes zurückgeführt, "qui dignatus est ostendere quid eiusmodi et tales servi de eius bonitate mereantur." 
Dic Vision des Celerinus zcigt, dass dieser Confessor im Hirten des Hermas lebte; wie diescm, so ist auch ihm die Kirche im Traum erschicnen?. In weleher Weise Gott aber den Befehl gegeben hat, den Numidicus z.u wählen, und wem, das verschweigt Cyprian. Die Gemeinde soll sich mit der blossen Thatsache begniggen 2.

4.

Cyprian war lïngst wieder in scine Gemeinde zurückgekehrt, hatte sic gesammelt, befestigt und das Schisma so gut wie beseitigt 3, da kam ihm plötzlich noch ein empfindlicher Angriff (im Jahre 253/54). Ein Confessor, uibrigens Laie, namens Florentius, erhob gegen ihn den schlimmsten Verdacht: nicht nur masslosen Hochmut warf er ihm brieflich vor und dass er cs sei, der die Gemeinde gespalten habe, sondern auch grobe, ja widernatürliche Unzucht4. Cyprian hat ihm im 66. Brief geantwortets; ob er sich von den Vorwürfen zu reinigen vermocht hat, das braucht uns hier nicht $z u$ kümmern. "Die Ausführungen überzeugen nicht ganz, jedoch der Schlusssatz: "habes tu litteras meas et ego tuas; in die iudicii ante tribunal Christi utrumque recitabitur" zeigt ein gutes Gewissen; aber lehrreich in dem uns interessierenden Zusammen-

I Mit Celerinus zusammen wird Aurelius (ep. 39,4) dem Clerus beigefügt. Auch von ihm heisst es, er sei "dizina dignatione promotus"; wie dies aber näher zu denken ist, darüber hat Cyprian geschwiegen.

2 Dass auch die karthaginiensische Gegenpartei (unter der Führung der Confessoren und einiger Presbyter) sich auf göttliche Offenbarung zu berufen hat, ist an sich wahrscheinlich oder vielmehr selbstverständlich, da ja, was der Confessor in religiösen Angelegenheiten thut, an und für sich als Anordnung Christi angeschant wurde. $\mathrm{Da}_{2}$ Cyprian dies in thesi nicht in Abrede stellen konnte und wollte, so schwieg er über dies Machtmittel seiner Gegner; aber die Warnungen im 43. Brief (c. 5) vor Pseudopropheten ist beachtenswert. Cyprian citiert Jerem 23, I6f.: „Nolite audire sermones pseudoprophetarum, quoniam visiones cordis corim frustrantur eos. locuntur, sed non ab ore domini. dicunt eis qui abiciunt verbum domini : pax erit vobis". Das war deutlich genug. Dann folgt das nicht minder anzügliche Citat Deut 13, 5 ; „et prophela ille aut somnizm somnians ille, qui locutus est, ut errare te faceret a domino deo tuo".

3 Auch dabei hat die Berufung auf Visionen nicht gefehlt. In dem Beschluss der karthaginiensischen Synode vom J. 252 wird die Wiederaufnahmen der Gefallenen (ep. 57, 5) motiviert (I) durch die Formel "spiritu suggerente“, (2) durch die andre "domino per visiones multas et manifestas admonente". Auch heisst es (l. c.) "divinitus frequenter nobis ostensum est", dass die herankommende neue Verfolgung schlimmer sein werde, als die decianische. Also der gesamte afrikanische Episcopat beruft sich auf Visionen!

4 Ep 66, I: „tam infanda tam turpia tam etiam gentilibus execranda“; c. I: "incestus“; c. 7: „,incesta, impia, nefanda“; c. 9: „domino et Christo eius puro atque immaculato ore sacrificia et in perscutione pariter et in pace indesinenter offero".

5 Der Charakter des Angreifers als Confessor legte dem Cyprian grosse Reserve auf. 
hang ist es, wie sich Cyprian für die Stellung des Bischofs, den niemand zu kritisieren habe ausser Gott x, auf eine göttliche Offenbarung beruft (c. 10):

„Memini quid iam mihi sit ostensum, immo quid sit servo obsequenti et timenti de dominica et divina auctoritate pracceptum; qui inter cetera quae ostendere et revelare dignatus est et hoc addidit: ,Itaque qui Christo non credit sacerdotem facienti postea credere incipiet sacerdotem vindicantic. quamquam sciam somnia ridicula et visiones ineptas quibusdam videri, sed utique illis qui malunt contra sacerdotes credere quam sacerdoti. sed nihil mirum, quando de Joseph fratres sui dixerunt: ,ecce somniator ille venit, nunc ergo venite, occidamus illum', et somniator postea quod somniaverat consecutus sit et occisores et venditores confusi sint, ut qui verbis prius non credidissent factis postmodum crederent".

Diese Ausführung ist in mehr als einer Hinsicht interessant: erstlich, die Offenbarung, auf die sich Cyprian beruft, ist ihm selbst geschenkt worden, daher auch hier wieder, wie oben sub I (Schluss), der Ausdruck der Bescheidenheit; zweitens, sie ist nicht ad hoc geschehen, sondern Cyprian erinnert sich eines früheren Vorgangs; drittens, das was er mitteilt, ist nur der Schluss dessen, was er als Offenbarung erlebt hat; viertens, es war keine blosse Schauung, sondern ein Befehl de dominica et divina auctoritate; fünftens, derselbe bezog sich auf die Würde des bischöflichen Amtes, war in ganz bestimmte Worte gefasst und drohte mit der Rache Christi wider den, der die Einsetzung des Bischofs, durch Christus in Zweifel zieht. Schon Ignatius hat, I40 Jahre vor Cyprian, den Geist verkündigen hören (ad Philad. 7): „Thut nichts ohne

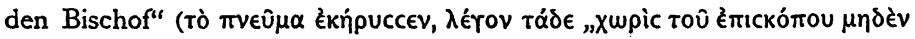

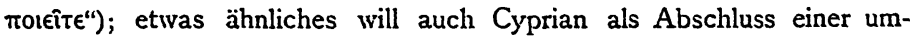
fassenderen, von ihm leider nicht mitgeteilten, Offenbarung gehört haben; nur enthielten die Worte eine furchtbare Drohung gegen die Verächter des Bischofs. Episkopalismus und Enthusiasmus haben sich in Cyprian enge verbunden, der. erstere ist bekanntlich in den Jahren $25 \mathrm{I}-54$ kräftig gewachsen, der letztere aber hat nicht abgenommen. Die beiden grossen Kräfte - die grössten, über die die Religion verfügt - liegen verbunden in Cyprian's Hand: die Gewalt des Amtes und die Gewalt des "Geistes". Wer kann diesen verbündeten Mächten widerstehen; sie müssen jeden Ungehorsam bändigen! Der Versuch aber, sie beide in dem Bischof zu vereinigen, den Cyprian gemacht hat, hat wenige oder keine Nachfolger in der Kirche gefunden. Alles hat seine

I Das widerspricht freilich seinen eigenen Ausführungen ep. 65 und 67 . 
Grenzen, und dieses Unternehmen, die Gewalten zu potenzieren, schien unglaubwirdig und liess sich nicht durchfuhren. Cyprian spricht in diesem Bricfe neben der Sprache des unverantwortlichen Bischofs die Sprache des Visionärs, der zu Christus ein Privatverhaltnis besitzt und in seinen Entschliussen direkt von "seinem Herrn" geleitet wird (c. 9: „prius dominum meum ${ }^{2}$ consulam an tibi pacem dari et te ad communicationem ccclesiae suac admitti sua ostensione et ad monitione permittal") ${ }^{2}$; allein cr selbst muss bereits klagen, „somnia ridicula ct visiones ineptas quibusdam videri". Er behauptet 2 war, so urtcilten nur dic, welche dem Bischof widerspenstig seien; aber es liegt auf der Hand, dass die Gemcinden auf die Dauer Bischöfe mit der "Amtsgnade", wie sie Cyprian in Anspruch nahm, nicht zu ertragen vermochten, wenn sie zugleich Propheten sein wollten $d$. h. lebendige göttliche Orakel. Dieser Zustand konnte erst eintreten, nachdem ein Bischof an die Spitze aller getreten und die Indifferenz zugleich so gross geworden war, dass man sich in der Sphäre der religio publica alles gefallen liess - also im 19. Jahrhundert. Cyprian ist durch seine Verbindung von Episkopalismus und Enthusiasmus sozusagen der erste Papst gewesen, und es hat lange gedauert, bis er einen Nachfolger erhalten hat ${ }^{3}$.

"Somnia ridicula, visiones ineptas" - dieses Urteil karthaginiensischer Christen bezeichnet eine grosse Wendung! Im Kampf mit dem Montanismus war man soweit noch nicht gegangen; man hatte die montanistischen Träume und Visionen nicht für lächerlich und absurd, sondern für diabolisch erklärt. Damit erkannte man sie noch immer als überirdisch an; erst die Lächerlichkeit tötet, und Christen in Karthago fingen an zu lachen.

5.

In Afrika war die Praxis, das Abendmahl mit Brod und Wasser zu feiern, ziemlich verbreitet und von langer Zeit her eingewurzelt. Be-

I Diese pietistische Sprache des Confessors oder Visionärs hatte Cyprian bislang niemals geführt.

2 In der Aufzählung seiner Rechtstitel als Bischof dem Cornelius von Rom gegenüber hat sich Cyprian (ep. 59,6) nicht auf seine Visionen berufen; das ist bemerkenswert.

3 Dass sich die Synoden als berufene Organe des h. Geistes fühlten, ist eine Erscheinung für sich und steht unter einer anderen Voraussetzung (musste auch andere, relativ erträglichere Folgen haben) als die persönliche Inspiration des Bischofs. Act. 15 bietet den verhängnisvollen Vers, auf, den die Inspiration der Synoden von Anfang an begründet worden ist. 
deutende Bischöfe, wie Cäcilius von Biltha, verteidigten dieselbe; man berief sich auf die Tradition und die Opportunität und hatte auch einen Schriftbeweis zur Hand. Cyprian nahm schweren Anstoss an dieser Praxis; aber er empfand die Schwierigkeit einzugreifen sehr lebhaft Bischöfe belehren zu wollen war ein gefahrvolles Unternehmen, aus dem leicht ein Schisma entspringen konnte. Endlich entschloss er sich zu einem öffentlichen Schritt in Form eines überaus sorgfalltig überlegten und ausgeführten Schreibens (ep. 63), dem weitere Schreiben nachfolgen sollten (s. ep. 63, 17). Vortrefliche Gründe standen ihm zu Gebote; aber augenscheinlich misstraute er ihrer durchschlagenden Kraft; darum berief er sich auch hier auf eine ihm zu Teil gewordene Specialanweisung Gottes. Gleich im Eingang des Briefs lesen wir (c. I):

"Non hoc putes, frater carissime, nos nostra et humana conscribere aut ultronea voluntate hoc nobis audaciter adsumere, cum mediocritatem nostram semper humili et vercunda moderatione teneamus. scd quando aliquid deo inspirante et mandanie praecipitur, necesse est domino serous fidilis obtemperet, excusatus apud omnes quod nihil sibi adroganter adsumat, qui ofiensam domini timere compellitur, nisi faciat quod iubetur".

Wie ihm Gott in diesem Fall die Inspiration und den Befehl hat zu Teil werden lassen, sagt er nicht; aber durch den Recurs auf einen solchen war er gegen den Vorwurf des Hochmuts bez. die Anklage, den massgebenden Oberbischof spielen zu wollen, gedeckt. Wer mag hier an die Inspiration Cyprians, d. h. an seinen Glauben an sie, glauben: Dass er aber ein starkes Pflichtgefühl mit Inspiration verwechselt hat, damit kann man ihn nicht entschuldigen. Augenscheinlich will er die Meinung erregen, Gott habe ihm in einer ganz bestimnten Weise durch eine Offenbarung den Auftrag gegeben, seiner Landeskirche mitzuteilen, das Abendmahl dürfe nicht länger mehr mit Brod und Wasser gefeiert werden.

\section{6.}

$\mathrm{Ob}$ Cyprian auch in dem Ketzertaufstreit sich auf eine Specialoffenbarung berufen hat, kann trotz ep. 73 , I 3 zweifelhaft erscheinen; denn die Worte, die er der Berufung auf eine irrtïmliche Tradition entgegensetzt: "post inspirationem et reculationem factam, qui in eo quod erraverat perseverat prudens et sciens, sine venia ignorantiae peccat".

I Man vgl dazu den Schluss des Briefs (c. 19), man müsse das, was in früherer Zeit irrtümlich geschehen war, domino monkeste nun corrigieren. 
brauchen nicht notwendig speziell auf den Ketzertaufstrcit bezogen zu werden, sondern können gencrell gemeint sein. Sehr bemerkenswert aber ist, dass Cyprian am Schluss des Briefs, von seinem Büchlein "de bono patientiac" sprechend, sagt, dass er dasselbe "permittente domino et inspirante" verfasst habe. Cyprian nimmt also für dieses Schriftwerk Autorisierung und Inspiration in Anspruch und erweist sich auch hierin als ein Christ, der cinen sehr alten, aber schon obsolet gewordenen Anspruch fortsetzt: die alte Überzeugung, dass jede erbauliche christliche Darlegung inspiricrt sei, war scit dem Ende des 2. Jalırhunderts nicht nur erschüttert, sondern untergraben und fast verschwunden. In der Folgezeit aber ist es sehr wichtig geworden, dass Cyprian selbst Inspiration für eine seiner erbaulichen Schriften ausdrücklich in Anspruch genommen hat; denn aus diesem Auspruch folgte, dass man im Abendland die cyprianischen Schriften ganz nahe an die heiligen Bücher herangerückt hat. Man vgl. wie sich bereits zu Lebzeiten Cyprian's die numidischen Con'essoren über jene Schriften ausgedrückt haben (ep. $77, \mathrm{I}$ ), und andere numidische Confessoren sprechen gleichzeitig (ep. 78, 2) in ihrem Brief an Cyprian von der "proplietia quam litteris tuis spiritu sancto plenus spopondisti."

7 .

Abgesehen von einer Stelle erinnere ich mich nicht, dass Cyprian in seinen Tractaten Visionen anführt und sie verwertet. Die einzige Stelle ist aber wertvoll, sie findet sich de mortalitate 19. 20. Erstlich erzählt hier Cyprian sehr ausführlich eine Vision, die ein Bischof auf dem Totenbett gehabt hat; zweitens spricht er selbst von häufigen Offenbarungen Gottes, die ihm selbst geschenkt worden sind:

„Cum quidam de collegis et consacerdotibus nostris infirmitate defessus et de adpropinquante morte sollicitus commeatum [Aufschub] sibi precaretur, adstitit deprecanti et iam paene morienti iuvenis honore et maiestate venerabilis, statu celsus et clarus aspectu et quem adstantem sibi vix possit humanus aspectus oculis carnalibus intueri, nisi quod talem videre iam poterat de saeculo recessurus. atque ille non sine quadam animi et vocis indignatione infremuit et dixit: ,Pati timetis, exire non vultis, quid faciam vobis?' increpantis vox et monentis, qui de persecutione sollicitus, de arcessitione securus non consentit ad praesens desiderium sed consulit in futurum". Cyprian bemerkt nun, dass diese Vision dem Sterbenden nicht um seinetwillen zu Teil geworden sei - 
er war ja schon im Verscheiden, - sondern unsretwegen: „audivit, ut diceret; audivit non sibi ille sed nobis". Dann fährt er fort:

„Nobis quoque ipsis minimis et extremis quotiens revelatum est, quam freqenter atque manifeste de dei dignatione pracceptum est, ut contestarer adsidue, ut publice praedicarem fratres nostros non esse lugendos arcessitione dominica de saeculo liberatos, cum sciamus non amitti sed praemitti, recedentes praecedere, ut proficiscentes ut navigantes solent desiderari eos debere, non plangi, nec accipiendas esse hic atras vestes, quando illi ibi indumenta alba iam sumpserint, occasionem dandam non esse gentilibus, ut nos merito ac iure reprehendant, quod quos vivere apud deum dicimus ut extinctos et perditos lugeamus etc.“.

Die Vision anlangend, so beachte man (cf. ep. II), dass Christus auch hier als der grosse, herrliche junge Mann erscheint; er zürnt wie in ep. II, und zwar ist der Unwille hier stärker ausgedrückt als dort. $\mathrm{Zu}$ den Worten: „vix possit humanus aspectus etc." vgl. Apoc. Petr. 6:

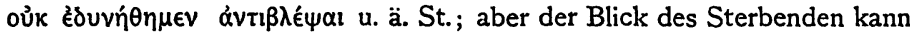
und darf schon sehen, was der noch ganz im Leben stehende nicht zu schauen vermag. Das Wort des Jünglings ist kraftvoll und prägt sich dem Gedächtnis tief ein. Wie erschütternd musste es wirken, als es der Sterbende erzählte und es nun weiter berichtet wurde! Es ist wiè ein Nachhall aus älterer Zeit; zu dem "pati timetis“ vgl. den Spruch des Parakleten bei Tertullian (de fuga 9; cf. de anima 55): „Nolite in lectulis nec in absorsibus et febribus optare exire, sed in martyriis, uti glorificetur qui est passus pro vobis". Seitdem ist die weltförmige Entwickelung fortgeschritten; die Christen sind nicht nur Martyriums-, sondern auch Todesscheu geworden. Neben dem Ernst liegt ein Hauch von Naivetät in dem Wort des Herrn: "Quid faciam vobis?" Es sind seine Erlösten; in die Hölle kann er sie nicht schicken; er will sie zu sich holen, aber sie fürchten sich zu leiden und sie wollen das Leben nicht verlassen! Beachtenswert ist es auch, dass ein $\mathrm{Bis}$ chof hier der Visionär ist; Cyprian ist also nicht der einzige Bischof gewesen, der damals Visionen in Afrika empfangen hat.

Dass ihm häufige Offenbarungen geschenkt worden sind - und zwar zu einem und demselben Zweck wiederholte -, sagt Cyprian nur hier. Wie sich dieselben vollzogen haben, hat er leider verschwiegen. Der Ausdruck äusserster Bescheidenheit (,nobis minimis et extremis") fehlt auch hier nicht, ebensowenig der terminus "dignatio“. Die Offenbarungen hatten den Zweck, auf Cyprian's Predigten einzuwirken; öffentlich sollte er gegen das Einreissen der heidnischen Sitte vorgehen, die 
Verstorbenen zu beklagen. Vermissen darf sie der Christ - mir scheint das schon eine gewisse Concession -; aber freuen soll er sich ihrer Reise. Wie stark müssen in dieser Gemeinde angesichts des Todes die Gefiulhle der Natur wiederum dic Oberherrschaft erlangt haben über dic religiöse Stimnung, wenn Cyprian es als einen speciellen göttlichen Auftrag mitteilt, man solle die Trauer und Trauerfeierlichkeiten in Bezug auf die Todten als unchristlich verbannen!

8.

Pontius, der Biograph Cyprian's, behauptet, wie Cyprian selbst (s. o.), dass die Flucht in der decianischen Verfolgung auf directen göttlichen Befehl erfolgt sei (Vita 7: "domino latebram tunc iubente", cf. c. 8: „secessum vere fuisse divinum"). Ausserdem erzählt er ausführlich eine "visitatio dei", welche Cyprian in seinem letzten Exil erlebt, und die der Bischof ihm und andern in seiner Ungebung berichtet habe. Es handelt sich um einen Traum, der nach den Worten Cyprians von Pontius wiedergegeben wird (c. 12).

„Apperuit mihi“, inquit, „nondum somni quiete sopito iuvenis ultra modum hominis enormis, qui cum me quasi ad praetorium duceret, videbar mihi tribunalis sedentis proconsulis admoneri etc". Die Fortsetzung ist nicht besonders interessant und mag nachgelesen werden; aber der ganze Vorgang bestätigt den Spott, der von einigen in der karthaginiensischen Gemeinde geäussert worden ist (s. o.); "Sehet den Träumer". Dass Jesus wiederum als junger Mann von ausserordentlicher Grösse erscheint, ist $\mathrm{zu}$ beachten.

Wie über Cyprian's "Enthusiasmus" zu urteilen ist, überlasse ich dem Leser; aber ich darf ihn wohl davor warnen, den Bischof für einen „heuchlerischen Pfaffen" zu erklären und unter dieses Urteil alles zu subsummieren. Gewiss hat es hier eine Linie gegeben, von welcher an der Enthusiasmus verschwand und die Politik eintrat; aber wo diese lag, hat Cyprian schwerlich selbst gewusst. Er hatte, wie das in der Antike üblich, heilige Träume; sie kamen öfters gerade dann, ,wenn er sie brauchte, und das übrige gab sich fast von selbst und noch einiges dazu.

Zum Schlusse stehe hier die Terminologie zusammen: das gewöhnliche waren bei Cyprian und anderen "visiones in noctibus" $d$. h. Träume (so werden sie geradezu bezeichnet); Visionen am Tage werden 


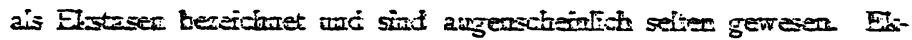

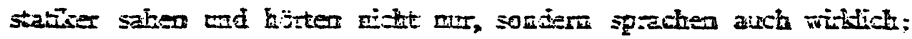

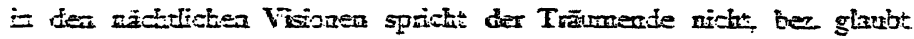
cIr wis sprecthen.

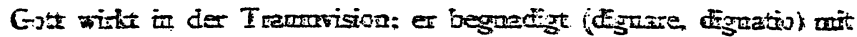
inz; sein Wirken hird als ostendere und revelare beschrieben; seine Absizhten dabei sind mannigfaltige finstruere, swadere, mandare, prascipire compellere, monere, admanera, hotari, exp-obare, castigare). Wieks wer

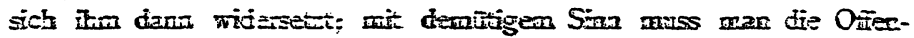
barrig empfangen ond ais serves obsequens et imens (servis iuderis) in gehorchen

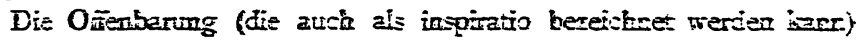
wird gexöjultch geschant (visio in strengen Sinok aber manchroal auch

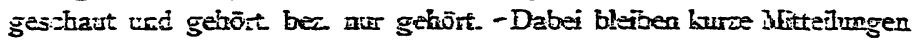
und Anweisungen im Gedāchtnis hatien. Wie die Orienbaruger ar deniker sinc, wo ledigiich Befehle ertent werden, bleib: dumkel

Ein angelus interpris escheint hin und her in den Orienbarugen, auch die Kirche (beides, jedenialls das zweite nach Hermas).

Wer auf Grund der ihm gewordenen Ofienbarugen spricht, spricht als Prophet uec bringt eine propheita; auch ganze Bïher bömen uxd düferi jetut noch als roa Gout inspiriert angesehen werien; freilich giebt es auch Pseuciopropheten und visiones corchs, die abzulehnen sind.

Arrdrücliche Befragungen dà Gotheit scheinea nicht stattgefunden zu hajen; doch hat sich Cyprian einmal zu der Aussage fortreissen lassen, er misse erst , seinen Herra befragen, ob er dies und dies inum dirfe. - Eine ekstatische Epidemie wird in dem Briefwechsel nur einmal erwähnt; die decianische Vertolgung war damals auf dem Höhepinfit; die Elstablker waren "pueri". 